\title{
LAS REDES DE DISTRIBUCIÓN INTELIGENTES Y EL BALANCE CARBONO NEUTRO COMO RETO PARA OPTIMIZAR EL CICLO DE CONSUMO ENERGÉTICO DE LAS CIUDADES
}

\author{
SMART DISTRIBUTION GRIDS AND NEUTRAL \\ CARBON BALANCE AS A CHALLENGE TO \\ OPTIMIZE ENERGY CYCLE IN CITIES
}

\section{Diana C. Valle Riestra Quevedo}

\section{Resumen}

El presente artículo analiza la relación entre el potencial de implementación de redes inteligentes y el ciclo de energía en las ciudades, ciclo que comprende la extracción, producción, distribución y consumo final de la energía como garante de desarrollo. Este interés se desprende de la constatación de que uno de los principales retos de las ciudades es paliar la escasez energética mediante tecnologías eficientes, capaces de reducir las emisiones de $\mathrm{CO}_{2}$. En relación a este requerimiento, el estudio se propone demostrar, en base a experiencias internacionales, que la manera inteligente de aplicar la tecnología en nuestras ciudades como respuesta al consumo energético es la adopción de tecnologías apropiadas y compatibles con cada realidad específica.

Palabras clave: Redes inteligentes; Balance cero; Ciclo de consumo energético.

\section{Abstract}

This article analyzes the relationship between the potential of smart grid implementation and the energy cycle in cities. A cycle that includes the extraction, production, distribution and final consumption of energy as a guarantor of 
development. This interest stems from the acknowledgment that one of the main challenges for cities is to reduce the effect of energy shortages through efficient technologies capable of reducing $\mathrm{CO} 2$ emissions. With regard to this requirement, the study aims to demonstrate, based on international experiences, that the smart way to apply technology in our cities in response to energy consumption is the adoption of appropriate technologies compatible with each specific reality.

Key words: Intelligent networks; Zero balance; Energy consumption cycle.

\section{INTRODUCCIÓN}

Uno de los principales retos de la vida en las ciudades es cómo aplicar soluciones inteligentes en el manejo de la infraestructura y equipamiento en que nos movemos, si como sociedad no alcanzamos aún el predominio de la racionalidad. Reflejo de esto son nuestras ciudades, convertidas en grandes núcleos de supuesto desarro1lo, pero sin una imagen objetiva hacia la cual direccionar esfuerzos.

La idea de desarrollo, modernidad y tecnología, como supuesto garante social de bienestar, se combinan en una idea confusa, cuyos principales efectos son la obsolescencia e ineficiencia de cuanto nos provee la ciudad y el flujo de energía que lo soporta. Como ecosistema tenemos una capacidad soporte finita de recursos para garantizar nuestra continuidad; sin embargo, hoy, al modelar nuestras ciudades, estamos perdiendo ese rasgo innato de entendimiento y respuesta al medio, dando margen a que la tecnología y su supuesta inteligencia, se posicione como única y necesaria respuesta.

Pero olvidamos algo, el discurso de la inteligencia tecnológica se fundamenta en lograr más con menos: menos consumo para mayor calidad de vida; pero esto se contrapone con el flujo de energía que requiere para su implantación. En el 2015, la huella generada por las TICs en la UE representaba el $10 \%$ de consumo eléctrico de una ciudad y el 4\% de emisiones de CO2. Frente a esta situación, se quiere identificar estos ámbitos de falla con las infraestructuras y principales ejes de intervención, a fin de proyectar una línea de pensamiento y estrategias de acción.

No es nuestro objetivo sustentar que debemos despojarnos de la tecnología, pues es fundamental en nuestro metabolismo urbano, sino orientarnos hacia un balance neutro de carbono, donde el consumo que requiere y lo que genere, sea considerado $\mathrm{CO} 2$ secuestrado, comparado con lo generado sin ella. El fin es la sostenibilidad desde el punto energético, funcional y social de las infraestructuras de nuestras ciudades.

\section{USO INTELIGENTE DE LAS TEC- NOLOGÍAS}

Una de las aplicaciones más interesantes de la tecnología, ambiciosa y a la vez polémica por integrar posturas contrarias, es la de la tecnología aplicada a escala ciudad para la gene- 
ración de Smart grids, o redes inteligentes, popularizada bajo la denominación de Smart City o Ciudad Inteligente. Bajo este concepto, se plantea a la tecnología como necesaria y única respuesta posible ante las falencias en cuanto a diseño e imagen de lo que se busca como "calidad de vida" en las ciudades, desencadenando un constante y creciente flujo de consumo de energía, pareciendo olvidar que la tierra posee límites biofísicos de los cuales dependemos.

Las tecnologías, en cualquier caso, deben usarse de manera inteligente, lo cual no significa dotar de más dispositivos, sino, por el contrario, hacer un uso racional de los mismos para lograr un equilibrio, evaluando qué tecnologías son las más óptimas para cada realidad y, en la medida de los posible, generando soluciones híbridas de tipos de energias empleadas y de sistemas pasivos y activos.

\subsection{El Rol de la Energía y los retos frente a su ciclo en las ciudades}

La globalización ha sido un catalizador del desarrollo económico de las ciudades, generando que grandes masas poblacionales enrumben en busca de un modelo de vida más avanzado, lo que redunda en un mayor coste de energía que lo sostenga; es decir, el crecimiento económico genera un crecimiento correlativo de la demanda, uno de cuyos pilares es la energía. La Agencia Internacional de Energía estima un incremento de $53 \%$ para el 2030, siendo responsables del 70\% de dicho incremento India y China. El uso de electricidad es una de las mayores fuentes indirectas de generación de $\mathrm{CO}_{2}$, por lo que representa un sector estratégico de acción. (IPCC, Panel de Expertos Cambio Climático, 2013).

Por tanto, considerando la energía como eje fundamental y a la vez crítico del desarrollo, se debe prever su planificación y vinculación al territorio buscando la eficiencia y el ahorro como tarea común. Es aquí donde las tecnologias inteligentes tienen un papel relevante desde una triple perspectiva, que involucra:

- Uso eficiente e inteligente de los recursos

- Desarrollo de tecnologías que ayudan a reducir el consumo

- Colaboración con el sector energético para mejorar la eficiencia de sus procesos

Es importante gestionar de manera eficiente todas las fases del ciclo energético. Para ello, la clave es analizar los DATOS, datos que en tiempo real nos den las métricas que permitan una detección temprana de las fallas, costos más reales de la energía y reducción de costes de mantenimiento.

Grandes empresas, como IBM, contemplan desde 1970 politicas de reducción de impactos de sus procesos en el medio ambiente, siendo lo más reciente su vinculación directa con la optimización de sus procesos y la generación de sus productos para la eficiencia y ahorro de energía. IBM es la primera empresa en alcanzar la ISO 14.001, por su Centro de Investigación de Ahorro Energético. Dentro 
de las acciones tomadas bajo la política de empresa está la compra de 96,000 MW/h en Certificados de energias Renovables para el 2006; así mismo, la generación de 13,700 MW/h de energía limpia a través de turbinas de viento, paneles solares fotovoltaicos y Biomasa. Estos números aún quedan cortos si lo comparamos con las métricas europeas, siendo resaltantes el caso del Reino Unido y sus 105,000 MW/h y Alemania con sus $11,800 \mathrm{MW} / \mathrm{h}$, equivalentes a 56,000 Tn $\mathrm{CO}_{2}$ eq.

Para ello, además de implementar soluciones en cuanto a sus procesos, genera dinámicas laborales como la concepción de E-Place, planteamiento relacionado con el espacio laboral, donde más de 100,000 trabajadores de IBM, desarrollan sus labores parcial o totalmente a distancia desde sus domicilios. Este porcentaje de trabajadores representa un ahorro de combustible estimado en un equivalente de 50,000 $\mathrm{TN} \mathrm{CO}_{2}$. Para ello, también es necesario un cambio de concepción espacial, siendo un buen ejemplo de esto el centro de sus oficinas Nor Este, Zurich. Este, logra ahorros entre el 25 y el 50\% correspondientes a electricidad, al dotar a sus espacios de flexibilidad y adaptabilidad a distintitas funciones, espacios libres, conectados y rotativos, pues no se asigna un espacio fijo a ningún trabajador. Un buen porcentaje de ellos permanecen en las mismas por periodos determinados; además, remplazan unidades fijas por computadores portátiles, reduciendo un consumo por ordenador de $200 \mathrm{~W}$ a 25W. El edificio está provisto también de sistemas de climatización optimizados, sistemas de placas fotovoltaicas generadoras de 40,000 $\mathrm{Kw} / \mathrm{h}$ y posee una envolvente con vidrio y sistemas automatizados de parasoles regulables, de acuerdo a las condiciones interiores y exteriores.

De igual manera, es importante considerar las grandes procesadoras de datos, conocidas también con las siglas CPDs. En un contexto como el actual, donde se procesan datos las 24 horas los 365 días del año, estos puntos neurálgicos deben ser optimizados para permitir la reducción del consumo que representan, entre $25 \%$ y 40\% de ahorro, porcentaje referido a su consumo eléctrico y su consumo por demanda de refrigeración. (Duart Belloque, 2007).

Como ejemplo de acciones concretas sobre estos centros de procesamiento, IBM plantea dos ejes importantes:

- Virtualización

- Soluciones tecnológicas especificas

Estas dos acciones, referidas al trabajo a distancia y a la concentración de los servicios, representan un desarrollo más racional de la topología de las redes, pues en lugar de tener distribuidos muchos puntos de procesamiento, todo lo concentran en un punto de gran capacidad de recepción, almacenamiento y procesamiento de datos. Un claro ejemplo de esto es que un servidor de IBM de última generación equivale a la capa- 
cidad de 150 racks de Intel; lo cual, traducido a cifras, representa un ahorro de 4,200 MW/h., logrado al reducir gastos de consumo eléctrico directos y demanda de refrigeración en varios puntos, concentrado solo en un espacio diseñado para esas necesidades, desde sus necesidades de refrigeración hasta la disposición de servidores, con el fin de optimizar su funcionamiento y extender su tiempo de vida.

Por tanto, ante estas cifras y la confirmación del rol importante de la tecnología en nuestro desarrollo como sociedad, es evidente la necesidad de encarar los retos de este sector, siendo los principales la reducción del gasto energético y el incremento de la eficiencia energética, para generar no solo disminución de la demanda, sino también la mejor calidad de distribución y oportunidad de extender su alcance.

Una primera conclusión a la que podemos llegar es que para poder planificar y gestionar la eficiencia energética y su principal objetivo vinculado el ahorro de la misma, es esencial fundamentar este ordenamiento mediante los datos; de tal manera que podemos afirmar que requerimos la relación directa del incremento de la velocidad para procesamiento, almacenamiento y análisis de los datos a fin de lograr un avance paralelo en la reducción del consumo de energía.

\subsection{Redes Inteligentes de distri- bución de energía}

Por lo antes analizado, se desprende que es fundamental la intervención de la tecnología, las IUN Intelligent Utilities Networks -un concepto a considerar-, haciendo referencia a las redes inteligentes desde la perspectiva de las telecomunicaciones como oportunidad y soporte para la eficiencia y generación de una Red de distribución inteligente de energía. Una red más inteligente que aprovecha la tecnología, al ser una red con capacidad para CONECTARSE. (Ortega, E. I., Cazco, D. A., Luna, V. O., \& Ortega, J. I. 2013)

Esta conexión es el eje clave para lograr:

a) Recolección automatizada de los datos.

b) Almacenamiento de grandes volúmenes de Data.

c) Herramientas de procesamiento y análisis.

Esto permitirá, en primer lugar, el uso óptimo de los activos con los que se cuenta en la red, y es una oportunidad para ampliar la misma a base de energías más limpias y de generación local, que se conviertan no en energía residual para la red pues es necesario salir de esa óptica mediante la incorporación de fuentes de energía renovables en la red, a través de puntos de desarrollo local, que tiene como real potencial realizar cambios en la matriz energética de una ciudad y estabilizar con ella las 
caídas de voltaje que generan pérdidas valiosas de información y de horas de producción y servicio en las ciudades.

\section{Gráfico $\mathbf{N}^{\circ} 1$.}

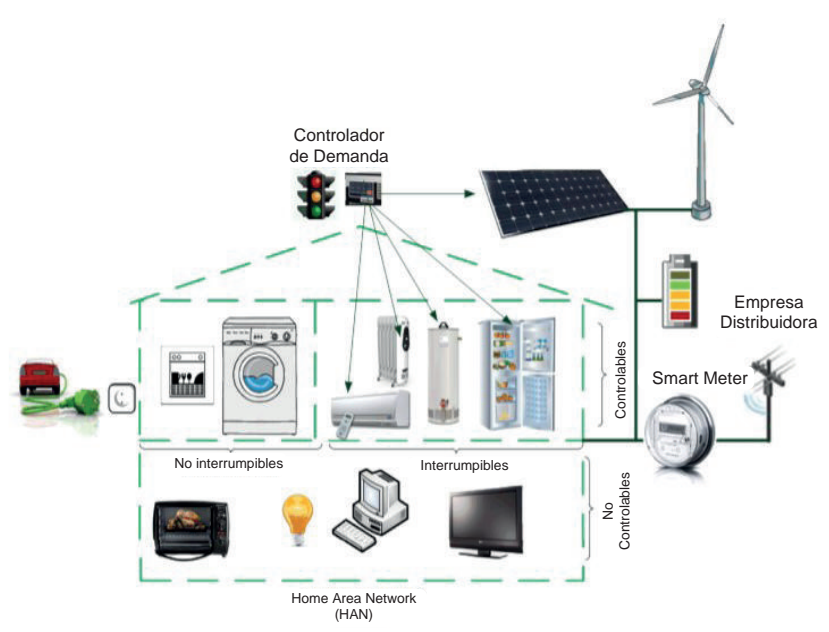

FUENTE: Ortega, E. I., Cazco, D. A., Luna, V. O., \& Ortega, J. I. (2013). Comunicaciones celulares para medición inteligente de energía eléctrica en sistemas de distribución. Ingenius, (10), 21-33.

Para ello, como podemos apreciar en el gráfico de referencia (Gráfico $\mathrm{N}^{\circ}$ 01), vemos que la clave de esta red es poder conectar equipos, sistemas de control, aplicaciones y sistemas de generación distribuida de energía a la red convencional.

Un papel clave para lograr la aplicabilidad de esto lo representan los Sistemas de Automatización de medición de consumo AMM. Estos sistemas permiten que cada punto se convierta en parte sustancial de la Red Inteligente. En un contexto como el de América Latina, donde la mayoría de la data con respecto a consumo eléctrico sigue dándose aún no de manera digital sino analógica, el reto es implementar estas tecnologias, siendo las principales ventajas de los medidores de consumo automatizados los siguientes:

- Monitoreo continuo de consumo de energía, por ejemplo, cada 15 minutos. Esto permite que los costos de facturación sean más reales y da la oportunidad al consumidor de ser más consiente y regular sus llamados periodos pico de consumo.

- Reducción de costos operativos de la red, lo cual libera recursos para poder optar por otras soluciones, como incorporación más activa de fuentes de energía renovables. 
Las redes de distribución inteligentes y el balance carbono neutro como reto para ...

- Información sobre consumo, el usuario deja de ser solo una fuente de Data para el sistema y se convierte en un actor bidireccional capas de modelar su consumo.

Para que esto se desarrolle y se logren los beneficios antes descritos, es necesario un marco Normativo y su respectivo Reglamento para su aplicabilidad, tal como, por ejemplo, ocurre en Ecuador, con el programa REDIE, Programa de Redes Inteligentes Ecuador. Este programa está enmarcado dentro de una serie de políticas de preocupación medio ambiental, siendo las principales: Plan Nacional del Buen Vivir del 2009 y las acciones del MEER, Ministerio de electricidad y Energías Renovables.

El gobierno ecuatoriano es consciente que este cambio producirá grandes resultados, pero debe darse de manera gradual, considerando el grado de madurez de las tecnologías y las necesidades específicas de su realidad. En nuestro país, por ejemplo, el Ministerio de Energía y Minas (MEN), a través de su Dirección de Eficiencia Energética, ha avanzado en cuanto a la reglamentación de Etiquetado energético de 09 artefactos de uso masivo, como el aire acondicionado y calderos. Sin embargo, aún no cuenta con un marco regulatorio aprobado para la generación distribuida a nivel nacional, aunque ya cuenta con un proyecto para la implementación desde el 2017 de seis (06) millones de medidores inteligentes a los largo del país. A pesar de que este proyecto no está aún aprobado, existen 05 empresas a nivel nacional que ya trabajan en la implementación de medidores inteligentes en edificaciones, que pretenden incorporarlo en su proyecto, para su futura incorporación a la red. (MEN, 2016)

Una variable poco analizada es la social, la cual libera a la tecnología de los rasgos de desconfianza de convertirla en un simple juego de monopolios privados en contextos poco desarrollados. En muchos países como el nuestro, desde la implantación de modelos económicos Neo Liberales, donde la energía es vista como un comercio y no es regulada su privatización, se ha producido el debilitamiento del control público y social, sobre todo por limitaciones al acceso a información. Cuando hablamos de esto, hacemos referencia a la Economía Social del Conocimiento (ESC), conocimiento y apertura al desarrollo del sector clave para la producción y crecimiento de las sociedades modernas. Solo a través del conocimiento es que la energía dejará de ser una mercancía y tomará su real dimensión de PROCOMUN, un producto para el bienestar y desarrollo común.

Los principales beneficios para nuestro país derivados de la transformación de la red eléctrica existente a una red inteligente, los podemos reducir en tres puntos:

1. Utilizar de manera masiva fuentes renovables y lograr la diversificación de la matriz energética, objetivo de acorde con el D.L. $\mathrm{N}^{\circ} 064$ 2010 EM.

2. Empoderamiento del consumidor, al democratizar la producción de energía. 
3. Gestión comunitaria de la Infraestructura.

Finalmente, esto es posible lograr ya que una red Inteligente es fundamentada en la tecnología de Telecomunicaciones repotenciada de una ciudad para permitir una BIDIRECCIONALIDAD de la red, entre el cliente y el consumidor, siendo repotenciado el rol del consumidor y convirtiéndose en un denominado Proconsumidor. En esto consiste el transito Bidireccional de la información para sistematizar la información y utilizar los recursos para resultados concretos y óptimos.

\section{Gráfico $\mathbf{N}^{\circ} 2$}

Elementos de la Red Urbana de Distribución Inteligente ELEMENTOS DE RED (SMART METERING)

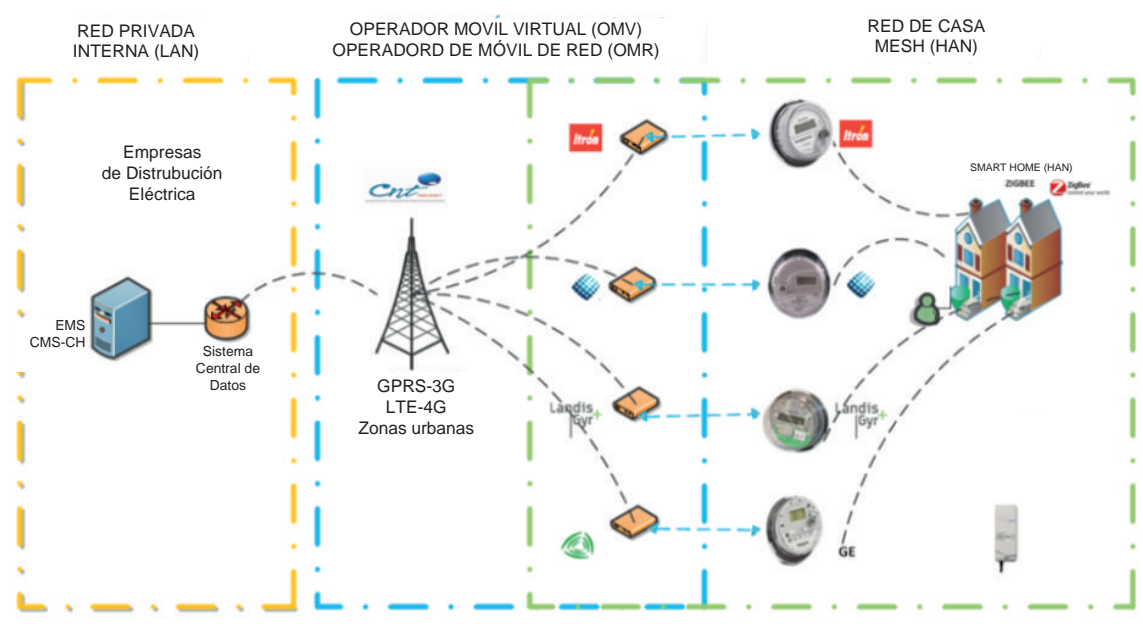

FUENTE: Ortega, E. I., Cazco, D. A., Luna, V. O., \& Ortega, J. I. (2013). Comunicaciones celulares para medición inteligente de energía eléctrica en sistemas de distribución. Ingenius, (10), 21-33.

\subsection{Balance de carbono neutro de las tecnologias en las ciudades}

Finalmente, como cierre de este artículo, se quiere estudiar cómo se planteó en primer lugar la incógnita sobre el real coste de la aplicación de estas tecnologías que buscan y pro- meten la eficiencia, pero que se fundamentan también en un consumo de energía y consecuente generación de $\mathrm{CO}_{2}$. Como informaba la Unión Europea, las TICS son responsables del 10\% de consumo eléctrico de una ciudad y de generar el 4\% de Emi- 
siones de CO2 (Díez Vázquez y otros, 2015: 241).

La Agencia Internacional de la Energía prevé que para el año 2020 habrá 50.000 millones de dispositivos conectados; solo en el 2013, el consumo de estos dispositivos fue alrededor de 616 TWh en el mundo, y, de este, un $80 \%$ se produjo en un estado de latencia. Así que la planificación de estas redes debe incluir métodos de minimización de consumo energético asociado o, caso contrario, considerar la energía consumida para su soporte carbono neutral a través del balance entre lo mitigado con respecto a lo que se generaría en su ausencia. Es decir, el consumo sería considerado neutral al ser menor del que se produciría de no intervenir en el ciclo de energía concreto. La Unión Europea tiene un proyecto interesante al respecto: el LIFE GREEN TIC, que trata de contrastar los efectos negativos de las TICs con acciones pilotos en los ámbitos administrativo, educativo y de gobernanza local, para que el balance de carbono de uso de las tecnologías sea neutro en una Ciudad Inteligente. (Información recuperada de: http://www.cece. gva.es/ite/docs/Buenas_Prac_Tic. pdf. 2015)

Para generar ese balance y lograr que el carbono sea considerado neutro, una red inteligente integrará la demanda y logrará la incorporación de Energías Renovables en la red. (Kemper, 2013).

Para ello, son importantes dos puntos:
- Comprensión del ámbito en las dimensiones territoriales y espaciales.

- Identificación de quiénes y cómo se consume la energía en el territorio, enfoque territorial que considere perspectivas tecnológicas y socioestructurales.

Esta modelación de los vectores energéticos se dará en dos modalidades: como energía integrada a la red existente, o como redes locales de energía autónoma, como es el caso de las islas Kythnos en Grecia. Algunos casos de éxito que podemos resaltar, son: Sistemas de generación distribuida a base de pequeñas Hidroeléctricas en Nepal; energias abiertas de generación eólica o red de Biodigestores de América Latina y el Carible. (Dafermos, G., Kotsampopoulos, P., Latoufis, K., Margaris, I., Washima, F. P., Ariza-Montobbio, P., \& López, J. 2015)

Se han identificado como final de este archivo las claves para lograr el balance energético de las redes distribuidas basadas en casos de éxito, como los mencionados a lo largo del estudio. Estos son:

a) Democratización de la producción de energía.

b) Inversión en la alfabetización energética.

c) Desarrollo conducido de la comunidad y participación del usuario para su empoderamiento.

d) Código abierto y tecnologías apropiadas y apropiables al entorno. 


\section{CONCLUSIONES}

Este tema, que no solo debe ser analizado sino estudiado desde distintas ópticas, como contribución para el incentivo de la creación de nuestro marco regulatorio nacional para aplicación de redes inteligentes. Nuestra propuesta considera que es importante entender el real aporte de la tec- b) Una red inteligente no es un lujo, ajeno a nuestro contexto, es una oportunidad real para lograr optimizar la distribución de la energía en un país donde esta no llega a cumplir la demanda. Esto, reduciendo sus costos de operatividad, los desperfectos de la distribución, mejorando su calidad, evitando las caídas de voltaje que impiden la eficiencia de equipos de alto rendimiento, y permitiendo la incorporación de generaciones alternativas que integren y estabilicen la red o que amplien la misma de manera segura a puntos donde no se accede $\mathrm{y}$, por ende, quedan excluidos del desarrollo que implica la energía.

c) Las tecnologías deben ser seleccionadas con criterios de sustentabilidad, es decir, ser apropiadas a cada contexto y no forzadas a modelos ajenos, y ser apropiables, o sea, tener códigos abiertos que permitan ser adoptadas por la comunidad de manera eficiente $\mathrm{y}$, de esta manera, reducir su margen de obsolescencia, que desmerece su rasgo de eficiencia. 
Las redes de distribución inteligentes y el balance carbono neutro como reto para ...

\section{BIBLIOGRAFÍA Y WEBGRAFÍA}

Alonso, A. (2011). Vitoria-Gasteiz Capital Verde Europea: Caminando hacia una ciudad competitiva neutra en carbono.

Dafermos, G., Kotsampopoulos, P., Latoufis, K., Margaris, I., Washima, F. P., Ariza-Montobbio, P., \& López, J. (2015). Energía: Conocimientos libres, energía distribuida y empoderamiento social para un cambio de matriz energética. In Buen Conocer-FLOK Society. Modelos sostenibles y politicas públicas para una economía social del conocimiento común y abierto en el Ecuador (pp. 431486). Asociación aLabs.

Diez Vázquez, J. A., Ruiz Tutor, J., Romero Tierno, C., Zubalez, N. (2015). "Reducir la huella de carbono de las ciudades inteligentes", I Congreso Ciudades Inteligentes, Madrid 24-25 Marzo, https://www.esmartcity.es/biblioteca/libro-decomunicaciones-i-congreso-ciudades-inteligentes (última consulta 07/07/2015, pág. 240).

Duart Belloque, V. (2007). Eficiencia y ahorro energético: contribución del sector de las tecnologías de la información. Universia Business Review, (13).

Fernández García, F. (2009). Ciudad y cambio climático: aspectos generales y aplicación al área metropolitana de Madrid.

Lorenzo, R. B., \& Rodríguez, B. A. L. SMART CITY Y GOBERNANZA: ELEMENTOS CATALIZADORES Y DESARROLLO DE LA DEMOCRACIA. EL CASO DE LA RED ESPAÑOLA DE CIUDADES INTELIGENTES.

Ortega, E. I., Cazco, D. A., Luna, V. O., \& Ortega, J. I. (2013). Comunicaciones celulares para medición inteligente de energía eléctrica en sistemas de distribución. Ingenius, (10), 21-33.

Paneque-de la Torre, Á., Aragonés-Beltrán, P., García-Melón, M., \& MartínezAlmela, J. (2011). Herramientas para la toma de decisiones aplicadas a la evaluación de los Planes de Acción Local del proyecto europeo RURENER. In Actas del XV Congreso Internacional de Ingeniería de Proyectos.

Rodríguez Bustamante, P. (2015). "Smart mobility o movilidad inteligente en España”, I Congreso Ciudades Inteligentes, Madrid 24-25 Marzo, https://www. esmartcity.es/biblioteca/libro-de-comunicaciones-i-congreso-ciudades-inteligentes (Última consulta 07/07/2015, pág. 171). 